\title{
Some Unified Finite Integrals involving Generalized Associated Legendre Polynomials
}

\author{
Vinita Agarwal \\ Department of Humanities \& Sciences, \\ Thakur College of Engineering \& Technology, Mumbai-400101, Maharashtra, India \\ Mukul Bhatt \\ Department of Humanities \& Sciences, \\ Thakur College of Engineering \& Technology, Mumbai-400101, Maharashtra, India \\ Mahesh Kumar Gupta \\ Department of Mathematics, M.S.J. Govt. P.G. College, Bharatpur-321001, Rajasthan
}

\begin{abstract}
In this paper four unified finite integrals are established. The first two of these integrals involve the product of a general class of a multivariable polynomials, $\bar{H}$ function and generalized associated Legendre function of second kind with arguments of the form $(x-a-1)^{-\frac{n}{2}}(x-a)^{\lambda-1}(b-x)^{\mu-1}(c x+d)^{\gamma}(g+x f)^{\delta}$. In third and fourth integrals general class of a multivariable polynomials are replaced with a generalized polynomial set in first and second integral. Some special cases and applications are also discussed. Since functions and polynomials occurring in these integrals are general in nature, these results provide interesting unifications and extensions of a large number of new and known results.
\end{abstract}

Keywords - $\bar{H}$ function, general class of a multivariable polynomials, generalized associated Legendre function, generalized polynomial set, fractional operator.

\section{INTRODUCTION}

A large number of integral formulae involving different types of special functions have been developed by many authors. Garg and Mittal [1] obtained an interesting unified integral involving Fox H-function. Considering the work of Garg and Mittal [1], Ali [2] gave three interesting unified integrals involving the hypergeometric function ${ }_{1} \mathrm{~F}_{2}$. By using Ali's method [2] Choi and Agarwal [3] presented two generalized integral formulas involving the Bessel function of the first kind, which are expressed in terms of the generalized (Wright) hypergeometric functions.

Agarwal [4] study some new unfied integral formulae associated with the $\bar{H}$-function. Each of these formulae involves a product of the $\bar{H}$-function and Srivastava polynomials with essentially arbitrary coefficients. They evaluated the formulae in terms of $\psi(z)$ [logarithmic derivative of $\Gamma(z)$ ]. Recently Chouhan and Khan [5] presents two new unified integral formulae involving the Fox H-function and M-Series. These results were expressed in terms of the $\mathrm{H}$ function.

\section{SOME DEFINITIONS}

A. Riemann-Liouville fractional calculus operator

The Riemann-Liouville fractional calculus operator of order $-\mu \square$ defined by Miller and Ross [6] as

${ }_{a} \mathrm{D}_{\mathrm{z}}^{-\mu}[f(\mathrm{z})]=\left\{\begin{array}{l}\frac{1}{\Gamma(\mu)} \int_{a}^{z}(z-t)^{\mu-1} f(t) \mathrm{dt}, \operatorname{Re}(\mu)>0 \\ \frac{\mathrm{d}^{m}}{\mathrm{~d} z^{m}}{ }_{a} \mathrm{D}_{z}^{-\mu-m}[f(\mathrm{z})], \quad-m<\operatorname{Re}(\mu) \leq 0, m \in \mathrm{N}\end{array}\right.$

where $m$ is a positive integer and the integral exists.

B. $\bar{H}$ - function

A more general function known as $\overline{\mathrm{H}}$-function was introduced by Inayat-Hussain [7] in the following form 


$$
\overline{\mathrm{H}}_{\mathrm{P}, \mathrm{Q}}^{\mathrm{M}, \mathrm{N}}[\mathrm{Z}]=\overline{\mathrm{H}}_{\mathrm{P}, \mathrm{Q}}^{\mathrm{M}, \mathrm{N}}\left[\mathrm{Z} \mid \begin{array}{l}
\left(a_{j}, \alpha_{j} ; A_{j}\right)_{1, N},\left(a_{j}, \alpha_{j}\right)_{\mathrm{N}+1, \mathrm{P}} \\
\left(b_{j}, \beta_{j}\right)_{1, \mathrm{M}},\left(b_{j}, \beta_{j} ; \mathrm{B}_{j}\right)_{\mathrm{M}+1, \mathrm{Q}}
\end{array}\right]=\frac{1}{2 \pi \mathrm{i}} \int_{-i \infty}^{i \infty} \bar{\phi}(\xi) z^{\xi} \mathrm{d} \xi
$$

Where $\quad \bar{\phi}(\xi)=\frac{\prod_{j=1}^{\mathrm{M}} \Gamma\left(b_{j}-\beta_{j} \xi\right) \prod_{j=1}^{\mathrm{N}}\left\{\Gamma\left(1-a_{j}+\alpha_{j} \xi\right)\right\}^{\mathrm{A}_{j}}}{\prod_{j=\mathrm{M}+1}^{\mathrm{Q}}\left\{\Gamma\left(1-b_{j}+\beta_{j} \xi\right)\right\}^{\mathrm{B}_{j}} \prod_{j=\mathrm{N}+1}^{\mathrm{P}} \Gamma\left(a_{j}-\alpha_{j} \xi\right)}$

and $i=\sqrt{-1}$. Here $a_{j} \quad(j=1, \ldots, \mathrm{P})$ and $b_{j} \quad(j=1, \ldots, \mathrm{Q})$ are complex parameters, $\alpha_{j} \geq 0(j=1, \ldots, \mathrm{P})$ and $\beta_{j} \geq 0(j=1, \ldots, \mathrm{Q})$ and the exponents $\mathrm{A}_{j}(j=1, \ldots, \mathrm{N})$ and $\mathrm{B}_{j}(j=\mathrm{N}+1, \ldots, \mathrm{Q})$ can take any non-integer values .

\section{Multivariable Polynomials}

The second general class of multivariable polynomials given by Srivastava [8] is defined and represented in the following modified form

$\mathrm{S}_{\mathrm{V}_{1}, \ldots, \mathrm{V}_{r}}^{\mathrm{U}_{1}, \ldots, \mathrm{U}_{r}}\left[x_{1}, \ldots, x_{r}\right]=\sum_{k_{1}=0}^{\left[\mathrm{V}_{1} / \mathrm{U}_{1}\right]} \ldots \sum_{k_{r}=0}^{\left[\mathrm{V}_{r} / \mathrm{U}_{r}\right]}(-\mathrm{V})_{\mathrm{U}_{1} k_{1}} \ldots\left(-\mathrm{V}_{r}\right)_{\mathrm{U}_{r} k_{r}} \cdot \mathrm{A}\left(\mathrm{V}_{1} k_{1} ; \ldots ; \mathrm{V}_{r} k_{r}\right) \frac{x_{1}^{k_{1}}}{k_{1} !} \ldots \frac{x_{r}^{k_{r}}}{k_{r} !}$

Where $\mathrm{V}_{i}=0,1,2, \ldots(i=1, \ldots, r), \mathrm{U}_{1}, \ldots, \mathrm{U}_{r}$ are arbitrary positive integers, the coefficients $\mathrm{A}\left[\mathrm{V}_{1}, k_{1} ; \ldots ; \mathrm{V}_{r}, k_{r}\right]$ being arbitrary constants, real or complex.

\section{Generalized Polynomial Set}

The generalized polynomial set $\mathrm{S}_{n}^{\alpha, \beta ; \tau}[x]$ is defined by the following Rodrigues type formula [9]

$\mathrm{S}_{\mathrm{N}}^{\alpha, \beta, \tau}[x]=(\mathrm{Ax}+\mathrm{B})^{-\alpha}\left(1-\tau x^{r}\right)^{\beta / \tau} \mathrm{T}_{\mathrm{k}, \ell}^{\mathrm{N}}\left[(\mathrm{A} x+\mathrm{B})^{\alpha+b \mathrm{~N}}\left(1-\tau x^{r}\right)^{\frac{\beta}{\tau}+\alpha \mathrm{N}}\right]$

with the differential operator being defined as

$$
\mathrm{T}_{k, \ell}=x^{\ell}\left(k+x \mathrm{D}_{x}\right)
$$

where $\mathrm{D}_{x} \equiv \mathrm{d} / \mathrm{d} x$

Raizada [9] presented $\mathrm{S}_{n}^{\alpha, \beta ; \tau}[x]$ in the following series form

$$
\mathrm{S}_{n}^{\alpha, \beta, \tau}[x]=\sum_{b_{1}, b_{2}, a_{1}, a_{2}} \theta\left(b_{1}, b_{2}, a_{1}, a_{2}\right) x^{\mathrm{R}}\left(1-\tau x^{t}\right)^{s n-a_{1}}
$$

Where

$$
\begin{aligned}
& \theta\left(b_{1}, b_{2}, a_{1}, a_{2}\right)= \frac{\mathrm{B}^{b n} \ell^{n}(-\tau)^{a_{1}}(-1)^{b_{1}}\left(-a_{1}\right)_{a_{2}}\left(-b_{1}\right)_{b_{2}}(\alpha)_{b_{1}}(-\alpha-b n)_{a_{2}}}{a_{1} ! a_{2} ! b_{1} ! b_{2} !\left(1-\alpha-b_{1}\right)_{b_{2}}}\left(\frac{-\beta}{\tau}-s n\right)_{a_{1}}\left(\frac{b_{2}+k+t a_{2}}{\ell}\right)_{n}\left(\frac{\mathrm{A}}{\mathrm{B}}\right)^{b_{1}} \\
& \mathrm{R}=\ell n+b_{1}+t a_{1} \\
& \sum_{b_{1}, b_{2}, a_{1}, a_{2}}=\sum_{a_{1}=0}^{n} \sum_{a_{2}=0}^{a_{1}} \sum_{b_{1}=0}^{n} \sum_{b_{2}=0}^{b_{1}}
\end{aligned}
$$

\section{E. Generalized Associated Legendre Polynomials}

Kuipers and Meulenbeld [10] introduced generalized associated Legendre functions $P_{k}^{m, n}(z), Q_{k}^{m, n}(z)$ of first and second kind respectively. 
The function $Q_{k}^{m, n}(z)$ can be presented in terms of hypergeometric function ${ }_{2} F_{1}(a, b ; c ; z)$ as $Q_{k}^{m, n}(z)=e^{m z i} 2^{k-\frac{m-n}{2}} \frac{\Gamma\left(k+\frac{m+n}{2}+1\right) \Gamma\left(k+\frac{m-n}{2}+1\right)}{\Gamma(2 k+2)}(z-1)^{-k-\frac{n}{2}-1}(z+1)^{\frac{n}{2}}{ }_{2} F_{1}\left(k-\frac{m-n}{2}+1, k+\frac{m+n}{2}+1 ; 2 k+2 ; \frac{2}{1-z}\right)$

\section{EXPERIMENT AND RESULT}

\section{A. First Integral}

$$
\begin{aligned}
& \int_{a}^{b} \frac{(x-a)^{\lambda-1}(b-x)^{\mu-1}(c x+d)^{\gamma}(g x+f)^{\delta}}{(x-a-1)^{\frac{n}{2}}} \mathrm{~S}_{\mathrm{v}_{1}, \ldots, v_{r}}^{\mathrm{U}_{1} \ldots \mathrm{v}_{r}}\left[y_{1} \frac{(x-a)^{\sigma_{1}}(b-x)^{\eta_{1}}}{(c x+d)^{\lambda_{1}}(g x+f)^{\mu_{1}}}, \ldots, y_{r} \frac{(x-a)^{\sigma_{r}}(b-x)^{\eta_{r}}}{(c x+d)^{\lambda_{r}}(g x+f)^{\mu_{r}}}\right] \\
& \overline{\mathrm{H}}_{\mathrm{P}, \mathrm{Q}}^{\mathrm{M}, \mathrm{N}}\left[\mathrm{z} \frac{(x-a)^{u}(b-x)^{v}}{(c x+d)^{p}(g x+f)^{q}}\right] Q_{k}^{m, n}\left(1-\frac{2}{x-a}\right) \mathrm{d} x \\
& =(b-a)^{\lambda+\mu+k}(a c+d)^{\gamma}(b g+f)^{\delta} e^{m \pi i} 2^{-\left(\frac{m-n}{2}+1\right)}(-1)^{-\left(k+\frac{n}{2}+1\right)} \Gamma\left(k+\frac{m-n}{2}+1\right) \\
& \sum_{\ell_{3}=0}^{\infty}(b-a)^{\ell_{3}} \frac{\left(k-\frac{m-n}{2}+1\right)_{\ell_{3}} \Gamma\left(k+\frac{m+n}{2}+1+\ell_{3}\right)}{\Gamma\left(2 k+2+\ell_{3}\right) \ell_{3} !} \sum_{k_{1}=0}^{\left[V_{1} / U_{1}\right]} \ldots \sum_{k_{r}=0}^{\left[V_{r} / U_{r}\right]}\left(-V_{1}\right)_{U_{1} k_{1}} \ldots\left(-V_{r}\right)_{U_{r} k_{r}} A\left[V_{1}, k_{1} ; \ldots ; V_{r}, k_{r}\right] \\
& \times \prod_{l=1}^{r} \frac{y_{l}^{k_{l}}(b-a)^{\left(\sigma_{l}+\eta_{l}\right) k_{l}}}{\left(k_{l} !\right)(a c+d)^{\lambda_{1} k_{l}}(b g+f)^{\mu_{l} k_{l}}} \sum_{\ell_{1}, \ell_{2}=0}^{\infty} \frac{1}{\ell_{1} ! \ell_{2} !}\left(\frac{c(a-b)}{a c+d}\right)^{\ell_{1}}\left(\frac{g(b-a)}{b g+f}\right)^{\ell_{2}}
\end{aligned}
$$

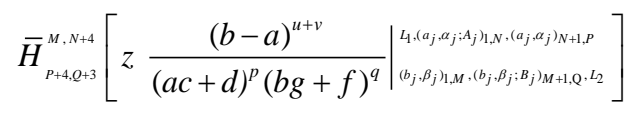

where

$$
\begin{aligned}
\mathrm{L}_{1}= & \left(1+\gamma-\ell_{1}-\Sigma \lambda_{l} k_{l}, p ; 1\right),\left(-\lambda-k-\ell_{1}-\ell_{3}-\Sigma \sigma_{l} k_{l}, u ; 1\right), \\
& \left(1+\delta-\ell_{2}-\Sigma u_{l} k_{l}, q ; 1\right),\left(1-\mu-\ell_{2}-\Sigma \eta_{l} k_{l}, v ; 1\right) \\
\mathrm{L}_{2}= & \left(1+\gamma-\Sigma \lambda_{l} k_{l}, p ; 1\right),\left(1+\delta-\Sigma \mu_{l} k_{l}, q ; 1\right), \\
& \left(-\lambda-\mu-\ell_{3}-k-\ell_{1}-\ell_{2}-\Sigma\left(\sigma_{l}+\eta_{l}\right) k_{l}, u+v ; 1\right)
\end{aligned}
$$

The conditions of validity of Eq (3.3) are

(a) $\quad \operatorname{Re}(\lambda, \mu)>0$

(b) $\quad \min \{u, v, p, q\} \geq 0$ (not all zero simultaneously)

(c) when $\min \left(\sigma_{l}, \eta_{l}\right) \geq 0 \quad(l=1, \ldots, r)$

$$
\begin{aligned}
& 1+\operatorname{Re}(\lambda+k)+u \min _{1 \leq j \leq \mathrm{M}} \operatorname{Re}\left(b_{j} / \beta_{j}\right)>0 \\
& \operatorname{Re}(\mu)+v \min _{1 \leq j \leq \mathrm{M}} \operatorname{Re}\left(b_{j} / \beta_{j}\right)>0 \\
& \text { When } \max \left(\sigma_{l}, \eta_{l}\right)<0(l=1, \ldots, r)
\end{aligned}
$$




$$
\begin{aligned}
& 1+\operatorname{Re}(\lambda+k)+\sum_{l=1}^{r}\left[\sigma_{l}\left(\frac{\mathrm{V}_{l}}{\mathrm{U}_{l}}\right)\right]+u \min _{1 \leq j \leq \mathrm{M}} \operatorname{Re}\left(b_{j} / \beta_{j}\right)>0 \\
& \operatorname{Re}(\mu)+\sum_{l=1}^{r}\left[\eta_{l}\left(\frac{\mathrm{V}_{l}}{\mathrm{U}_{l}}\right)\right]+v \min _{1 \leq j \leq \mathrm{M}} \operatorname{Re}\left(b_{j} / \beta_{j}\right)>0
\end{aligned}
$$

When $\sigma_{l} \geq 0, \eta_{l}<0,(l=1, \ldots, r),(\mathrm{I})$ and $(\mathrm{IV})$ are satisfied.

When $\eta_{l} \geq 0, \sigma_{l}<0, \quad(l=1, \ldots, r)$, (II) and (III) are satisfied.

$$
\max \left\{\left|\frac{c(b-a)}{a c+d}\right|,\left|\frac{g(b-a)}{b g+f}\right|\right\}<1, b \neq a \text {. }
$$

(e) If $k+\frac{m+n}{2} \neq-1,-2, \ldots ; k \pm \frac{m-n}{2} \neq 0, \pm 1, \pm 2, \ldots ; 2 k+2 \neq 0,-1,-2, \ldots \quad|z-1|>2$

PROOF. To evaluate the integral, $\mathrm{S}_{\mathrm{v}_{1}, \ldots, \mathrm{v}_{r}}^{\mathrm{U}_{1}, \ldots, \mathrm{U}_{r}}[x]$ is replaced by its series representation using equation Eq (2.4), $\overline{\mathrm{H}}$ function is replaced by its Mellin-Barnes contour integral form using Eq (2.2) and $Q_{k}^{m, n}(z)$ is replaced by its hypergeometric function form using $\mathrm{Eq}(2.10)$ in the left hand side of $\mathrm{Eq}(3.1)$. Then the powers of $(x-a)$, $(b-x)$, $(c x+d)$ and $(g x+f)$ are collected. In the resulting expression the order of integration and summation is interchanged (which is permissible under the conditions stated with (3.1)). The powers of $(c x+d)$ and $(g x+f)$ are simplified by applying following binomial expansions for $x \in[a, b]$

$$
\begin{aligned}
& (c x+d)^{m}=(a c+d)^{m} \sum_{\ell_{1}=0}^{\infty} \frac{(-m)_{\ell_{1}}}{\ell_{1} !}\left\{\frac{-c(x-a)}{a c+d}\right\}^{\ell_{1}},|(x-a) c|<|a c+d| \\
& (g x+f)^{n}=(b g+f)^{n} \sum_{\ell_{2}=0}^{\infty} \frac{(-n)_{\ell_{2}}}{\ell_{2} !}\left\{\frac{g(b-x)}{b g+f}\right\}^{\ell_{2}},|g(b-x)|<|b g+f|
\end{aligned}
$$

and the innermost integral is solved with the help of the following Eulerian type integral

$$
\int_{a}^{b}(x-a)^{\lambda-1}(b-x)^{\mu-1} \mathrm{~d} x=(b-a)^{\lambda+\mu-1} \mathrm{~B}(\lambda, \mu)
$$

Where $\operatorname{Re}(\lambda)>0, \operatorname{Re}(\mu)>0, b \neq a$ and $\mathrm{B}(\lambda, \mu)$ represents beta function

The beta function is simplified in terms of gamma function and resulting Mellin-Barnes contour integral is interpreted as $\overline{\mathrm{H}}$-function. After little simplification, the right hand side of Eq (3.1) is obtained.

B. Second Integral

$$
\begin{aligned}
& \int_{a}^{b} \frac{(x-a)^{\lambda-1}(b-x)^{\mu-1}}{(c x+d)^{\gamma}(g x+f)^{\delta}(x-a-1)^{\frac{n}{2}}} S_{V_{1}, \ldots, v_{r}}^{U_{1}, U_{r}}\left[y_{1} \frac{(c x+d)^{\lambda_{1}}(g x+f)^{\mu_{1}}}{(x-a)^{\sigma_{1}}(b-x)^{\eta_{1}}}, \ldots, y_{r} \frac{(c x+d)^{\lambda_{r}}(g x+f)^{\mu_{r}}}{(x-a)^{\sigma_{r}}(b-x)^{\eta_{r}}}\right] \\
& \times \bar{H}_{P, Q}^{M, N}\left[z \frac{(c x+d)^{p}(g x+f)^{q}}{(x-a)^{u}(b-x)^{v}}\right] Q_{k}^{m, n}\left(1-\frac{2}{x-a}\right) d x \\
& =(b-a)^{\lambda+\mu+k}(a c+d)^{\gamma}(b g+f)^{\delta} e^{m \pi i} 2^{-\left(\frac{m-n}{2}+1\right)}(-1)^{-\left(k+\frac{n}{2}+1\right)} \Gamma\left(k+\frac{m-n}{2}+1\right) \\
& \sum_{\ell_{3}=0}^{\infty}(b-a)^{\ell_{3}} \frac{\left(k-\frac{m-n}{2}+1\right)_{\ell_{3}} \Gamma\left(k+\frac{m+n}{2}+1+\ell_{3}\right)}{\Gamma\left(2 k+2+\ell_{3}\right) \ell_{3} !} \sum_{k_{1}=0}^{\left[V_{1} / U_{1}\right]} \ldots \sum_{k_{r}=0}^{\left[V_{r} / U_{r}\right]}\left(-V_{1}\right)_{U_{1} k_{1} \ldots} \ldots\left(-V_{r}\right)_{U_{r} k_{r}} A\left[V_{1}, k_{1} ; \ldots ; V_{r}, k_{r}\right]
\end{aligned}
$$




$$
\begin{aligned}
& \times \prod_{l=1}^{r} \frac{y_{l}^{k_{l}}(b-a)^{\left(\sigma_{l}+\eta_{l}\right)^{k_{l}}}}{\left(k_{l} !\right)(a c+d)^{\lambda_{l} k_{l}}(b g+f)^{\mu_{l} k_{l}}} \sum_{\ell_{1}, \ell_{2}=0}^{\infty} \frac{1}{\ell_{1} ! \ell_{2} !}\left(\frac{c(a-b)}{a c+d}\right)^{\ell_{1}}\left(\frac{g(b-a)}{b g+f}\right)^{\ell_{2}} \\
& \times \bar{H}_{P+3, Q+4}^{M+4, N}\left[z \frac{(a c+d)^{p}(b g+f)^{q}}{(b-a)^{u+v}} \mid \begin{array}{l}
\left(a_{j}, \alpha_{j} ; A_{j}\right)_{1, N},\left(a_{j}, \alpha_{j}\right)_{N+1, P,}, L_{1}^{*} \\
L_{2}^{*},\left(b_{j}, \beta_{j}\right)_{1, M},\left(b_{j}, \beta_{j} ; B_{j}\right)_{M+1, Q}
\end{array}\right]
\end{aligned}
$$

where

$$
\begin{aligned}
& L_{1}^{*}=\left(\gamma-\Sigma \lambda_{\ell} k_{\ell}, p\right),\left(\delta-\Sigma \mu_{\ell} k_{\ell}, q\right),\left(1+\lambda+\mu+k+\ell_{1}+\ell_{2}+\ell_{3}-\Sigma\left(\sigma_{\ell}+\eta_{\ell}\right) k_{\ell}, u+v\right) \\
& L_{2}^{*}=\left(\gamma+\ell_{1}-\Sigma \lambda_{\ell} k_{\ell}, p\right),\left(\delta+\ell_{2}-\Sigma \mu_{\ell} k_{\ell}, q\right),\left(1+\lambda+\ell_{1}+\ell_{3}+k-\Sigma \sigma_{\ell} k_{\ell}, u\right), \quad\left(\mu+\ell_{2}-\Sigma \eta_{\ell} k_{\ell}, v\right)
\end{aligned}
$$

The conditions of validity of Eq (3.7) are

(a) $\quad \operatorname{Re}(\lambda, \mu)>0$

(b) $\min \{u, v, p, q\} \geq 0$ (not all zero simultaneously)

(c) When $\max \left(\sigma_{\ell}, \eta_{\ell}\right)<0(\ell=1, \ldots, r)$

$$
\begin{aligned}
& 1+\operatorname{Re}(\lambda+k)-u \max _{1 \leq j \leq \mathrm{N}} \operatorname{Re}\left(\frac{a_{j}-1}{\alpha_{j}}\right)>0 \\
& \operatorname{Re}(\mu)-v \max _{1 \leq j \leq \mathrm{N}} \operatorname{Re}\left(\frac{a_{j}-1}{\alpha_{j}}\right)>0
\end{aligned}
$$

When $\min \left(\sigma_{\ell}, \eta_{\ell}\right) \geq 0(\ell=1, \ldots, r)$

$$
\begin{aligned}
& 1+\operatorname{Re}(\lambda+k)-\sum_{\ell=1}^{r}\left[\sigma_{\ell}\left(\frac{V_{\ell}}{U_{\ell}}\right)\right]+u \max _{1 \leq j \leq N} \operatorname{Re}\left(\frac{a_{j}-1}{\alpha_{j}}\right)>0 \\
& \operatorname{Re}(\mu)-\sum_{\ell=1}^{r}\left[\eta_{\ell}\left(\frac{\mathrm{V}_{\ell}}{\mathrm{U}_{\ell}}\right)\right]+v \max _{1 \leq j \leq \mathrm{N}} \operatorname{Re}\left(\frac{a_{j}-1}{\alpha_{j}}\right)>0
\end{aligned}
$$

When $\sigma_{\ell} \geq 0, \eta_{\ell}<0 \quad(\ell=1, \ldots, r)$, (V) and (VII) are satisfied.

When $\eta_{\ell} \geq 0, \sigma_{\ell}<0(\ell=1, \ldots, r)$, (VI) and (VIII) are satisfied.

(d) $\max \left\{\left|\frac{(b-a) c}{a c+d}\right|,\left|\frac{(b-a) g}{b g+f}\right|\right\}<1 ; b \neq a$.

(e) If $k+\frac{m+n}{2} \neq-1,-2, \ldots ; k \pm \frac{m-n}{2} \neq 0, \pm 1, \pm 2, \ldots ; 2 k+2 \neq 0,-1,-2, \ldots, \quad|z-1|>2$

Proof: The integral (3.7) can be evaluated in a similar way as that of the first integral.

\section{Third Integral}

$$
\begin{aligned}
& \int_{a}^{b} \frac{(x-a)^{\lambda-1}(b-x)^{\mu-1}(c x+d)^{\gamma}(g x+f)^{\delta}}{(x-a-1)^{\frac{n}{2}}} S_{n}^{\alpha, \beta, z}\left[y \frac{(x-a)^{\sigma}(b-x)^{\eta}}{(c x+d)^{\zeta}(g x+f)^{v}}\right] \\
& \times \bar{H}_{P, Q}^{M, N}\left[z \frac{(x-a)^{u}(b-x)^{v}}{(c x+d)^{p}(g x+f)^{q}}\right] Q_{k}^{m, n}\left(1-\frac{2}{x-a}\right) d x \\
& =(b-a)^{\lambda+k+\mu}(a c+d)^{\gamma}(b g+f)^{\delta} e^{m \pi i} 2^{-\left(\frac{m-n}{2}+1\right)}(-1)^{-\left(k+\frac{n}{2}+1\right)} \Gamma\left(k+\frac{m-n}{2}+1\right) \\
& \quad \times \sum_{b_{1}, b_{2}, a_{1}, a_{2} \ell_{1}, \ell_{2}, \ell_{3}, \ell_{4}=0} \frac{\left(k-\frac{m-n}{2}+1\right)_{\ell_{3}} \Gamma\left(k+\frac{m+n}{2}+1+\ell_{3}\right) \tau^{\ell_{1}} y^{R+\ell_{1} t}\left(a_{1}-s n\right)_{\ell_{1}}}{\ell_{1} ! \ell_{2} ! \ell_{3} ! \ell_{4} !} \\
& \frac{(b-a)^{\ell_{3}+\left(R+\ell_{1}\right)(\sigma+\eta)} \theta\left(b_{1}, b_{2}, a_{1}, a_{2}\right)}{(a c+d)^{\left(R+\ell_{1}\right) \zeta}(b g+f)^{\left(R+t \ell_{1}\right) v}}\left(\frac{c(a-b)}{a c+d}\right)^{\ell_{2}}\left(\frac{g(b-a)}{b g+f}\right)^{\ell_{4}} \bar{H}_{P+4, Q+3}^{M, N+4}\left[\left.z \frac{(b-a)^{u+v}}{(a c+d)^{p}(b g+f)^{q}}\right|_{\left(b_{j}, \beta_{j}\right)_{1, M},\left(b_{j}, \beta_{j} ; B_{j}\right)_{M+1, Q}, L_{4}} ^{L_{3},\left(a_{j}, \alpha_{j} ; A_{j}\right)_{1, N},\left(a_{j}, \alpha_{j}\right)_{N+1, P}}\right]
\end{aligned}
$$


where

$$
\begin{aligned}
L_{3}= & \left(1+\gamma-\ell_{2}-\left(R+t \ell_{1}\right) \zeta, p ; 1\right),\left(1+\delta-\ell_{4}-\left(R+t \ell_{1}\right) v, q ; 1\right), \\
& \left(-\lambda-k-\ell_{3}-\ell_{2}-\left(R+t \ell_{1}\right) \sigma, u ; 1\right),\left(1-\mu-\ell_{4}-\left(R+t \ell_{1}\right) \eta, v ; 1\right) \\
L_{4}= & \left(1+\gamma-\left(R+t \ell_{1}\right) \zeta, p ; 1\right),\left(1+\delta-\left(R+t \ell_{1}\right) v, q ; 1\right), \\
& \left(-\lambda-\mu-k-\left(\ell_{2}+\ell_{3}+\ell_{4}\right)-\left(R+t \ell_{1}\right)(\sigma+\eta), u+v ; 1\right)
\end{aligned}
$$

The conditions of validity of Eq (3.10) are

(a) $\quad \operatorname{Re}(\lambda, \mu)>0$

(b) $\min \{\sigma, \eta, \zeta, v, u, v, p, q\} \geq 0$ (not all zero simultaneously)

(c) $\quad 1+\operatorname{Re}(\lambda+k)+\operatorname{Re}(\sigma)+u \min _{1 \leq j \leq \mathrm{M}} \operatorname{Re}\left(b_{j} / \beta_{j}\right)>0$

$$
\operatorname{Re}(\mu)+\operatorname{Re}(\eta)+v \min _{1 \leq j \leq \mathrm{M}} \operatorname{Re}\left(b_{j} / \beta_{j}\right)>0
$$

(d) $\quad \max \left\{\left|\frac{c(b-a)}{a c+d}\right|,\left|\frac{g(b-a)}{b g+\mathrm{f}}\right|\right\}<1, b \neq a$.

(e) If $k+\frac{m+n}{2} \neq-1,-2, \ldots ; k \pm \frac{m-n}{2} \neq 0, \pm 1, \pm 2, \ldots ; \quad 2 k+2 \neq 0,-1,-2, \ldots$

$$
|z-1|>2
$$

PROOF. To evaluate the integral, the generalized polynomial set $S_{n}^{\alpha, \beta, \tau}[z]$ is replaced by its series representation form using Eq(2.6), $\overline{\mathrm{H}}$-function is replaced by its Mellin-Barnes contour integral form using Eq(2.2)and $Q_{k}^{m, n}(z)$ is replaced by its hypergeometric function form using $\mathrm{Eq}(2.10)$ in the left hand side of $\mathrm{Eq(3.10)}$. Then the powers of $(x-a),(b-x),(c x+d)$ and $(g x+f)$ are collected. In the resulting expression the order of integration and summation is interchanged (which is permissible under the conditions stated with (3.3)). The integral is simplified using Eqs (3.4), (3.5) and (3.6) .

The beta function is simplified in terms of gamma function and resulting Mellin-Barnes contour integral is interpreted as $\overline{\mathrm{H}}$-function. After little simplification the right hand side of $\mathrm{Eq}(3.10)$ is obtained.

D. Fourth Integral

$$
\begin{aligned}
& \int_{a}^{b} \frac{(x-a)^{\lambda-1}(b-x)^{\mu-1}}{(c x+d)^{\gamma}(g x+f)^{\delta}(x-a-1)^{\frac{n}{2}}} S_{n}^{\alpha, \beta, r}\left[y \frac{(c x+d)^{\zeta}(g x+f)^{\nu}}{(x-a)^{\sigma}(b-x)^{\eta}}\right] \\
& \times \bar{H}_{P, Q}^{M, N}\left[z \frac{(c x+d)^{p}(g x+f)^{q}}{(x-a)^{u}(b-x)^{v}}\right] Q_{k}^{m, n}\left(1-\frac{2}{x-a}\right) d x \\
& =(b-a)^{\lambda+k+\mu}(a c+d)^{\gamma}(b g+f)^{\delta} e^{m \pi i} 2^{-\left(\frac{m-n}{2}+1\right)}(-1)^{-\left(k+\frac{n}{2}+1\right)} \Gamma\left(k+\frac{m-n}{2}+1\right) \\
& \sum_{b_{1}, b_{2}, a_{1}, a_{2} \ell_{1}, \ell_{2}, \ell_{3}, \ell_{4}=0} \frac{\left(k-\frac{m-n}{2}+1\right)_{\ell_{3}} \Gamma\left(k+\frac{m+n}{2}+1+\ell_{3}\right)}{\Gamma\left(2 k+2+\ell_{3}\right)} \frac{\tau^{\ell_{1}} y^{R+\ell_{1} t}\left(a_{1}-s n\right)_{\ell_{1}}}{\ell_{1} ! \ell_{2} ! \ell_{3} ! \ell_{4} !} \\
& \times \frac{(a c+d)^{\left(R+t \ell_{1}\right) \zeta}(b g+f)^{\left(R+\ell_{1}\right) v} \theta\left(b_{1}, b_{2}, a_{1}, a_{2}\right)}{(b-a)^{\left(R+t \ell_{1}\right)(\sigma+\eta)-\ell_{3}}}\left(\frac{c(a-b)}{a c+d}\right)^{\ell_{2}}\left(\frac{g(b-a)}{b g+f}\right)^{\ell_{4}}
\end{aligned}
$$




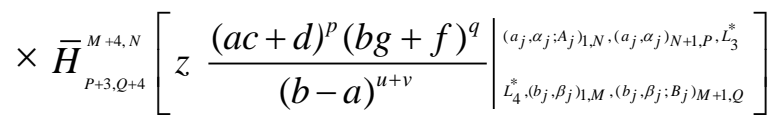

where

$L_{3}^{*}=\left(\gamma-\left(R+t \ell_{1}\right) \zeta, p\right),\left(\delta-\left(R+t \ell_{1}\right) v, q\right), \quad\left(1+\lambda+\mu+k+\ell_{2}+\ell_{3}+\ell_{4}-\left(R+t \ell_{1}\right)(\sigma+\eta), u+v\right)$

$L_{4}^{*}=\left(\gamma+\ell_{2}-\left(R+t \ell_{1}\right) \zeta, p\right),\left(\delta+\ell_{4}-\left(R+t \ell_{1}\right) v, q\right),\left(1+\lambda+k+\ell_{2}+\ell_{3}-\left(R+t \ell_{1}\right) \sigma, u\right),\left(\mu+\ell_{4}-\left(R+t \ell_{1}\right) \eta, v\right)$,

and $\theta\left(b_{1}, b_{2}, a_{1}, a_{2}\right), R$ and $\sum_{b_{1}, b_{2}, a_{1}, a_{2}}$ are as given in Eqs (2.7), (2.8) and (2.9) respectively.

The conditions of validity for (3.13) are

(a) $\operatorname{Re}(\lambda, \mu)>0$

(b) $\quad \min \{\sigma, \eta, \zeta, v, u, v, p, q\} \geq 0$ (not all zero simultaneously)

(c) $\quad 1+\operatorname{Re}(\lambda+k)-\operatorname{R} \sigma-u \max _{1 \leq j \leq \mathrm{N}} \operatorname{Re}\left(\frac{a_{j}-1}{\alpha_{j}}\right)>0$

$\operatorname{Re}(\mu)-\operatorname{Re}(\eta)-v \max _{1 \leq j \leq \mathrm{N}} \operatorname{Re}\left(\frac{a_{j}-1}{\alpha_{j}}\right)>0$

(d)

$$
\max \left\{\left|\frac{c(b-a)}{a c+d}\right|,\left|\frac{g(b-a)}{b g+f}\right|\right\}<1, b \neq a .
$$

(e) If $k+\frac{m+n}{2} \neq-1,-2, \ldots ; k \pm \frac{m-n}{2} \neq 0, \pm 1, \pm 2, \ldots ; 2 k+2 \neq 0,-1,-2, \ldots$

$$
|z-1|>2
$$

PROOF. The integral (3.13) can be evaluated in a similar way as that of the third integral.

E. Applications

The results obtained from these integrals can be applied to obtain Riemann-Liouville fractional calculus operator of unified functions. Some of the examples are shown below.

(i) Taking $b=z, \eta=v=0$ in $\mathrm{Eq}(3.1)$, the Riemann-Liouville fractional calculus operator of order $-\mu$ of a unified function is obtained as

$$
\begin{aligned}
& { }_{a} \mathrm{D}_{z}^{-\mu}\left\{\frac { ( z - a ) ^ { \lambda - 1 } ( c z + d ) ^ { \gamma } ( g + z f ) ^ { \delta } } { ( z - a - 1 ) ^ { \frac { n } { 2 } } } \mathrm { S } _ { \mathrm { V } _ { 1 } , \ldots , \mathrm { V } _ { r } } ^ { \mathrm { U } _ { 1 } , \ldots , \mathrm { U } _ { r } } \left[y_{1} \frac{(z-a)^{\sigma_{1}}}{(c z+d)^{\lambda_{1}}(g z+f)^{\mu_{1}}, \ldots,}\right.\right. \\
& \left.\left.y_{r} \frac{(z-a)^{\sigma_{r}}}{(c z+d)^{\lambda_{r}}(g z+f)^{\mu_{r}}}\right] \overline{\mathrm{H}}_{\mathrm{P}, \mathrm{Q}, \mathrm{N}}^{\mathrm{M}, \mathrm{N}}\left[\frac{z^{*}(z-a)^{u}}{(c z+d)^{p}(g z+f)^{q}}\right] Q_{k}^{m, n}\left(1-\frac{2}{z-a}\right)\right\} \\
& =e^{m \pi i} 2^{-\left(\frac{m-n}{2}+1\right)}(-1)^{-\left(k+\frac{n}{2}+1\right)} \Gamma\left(k+\frac{m-n}{2}+1\right) \Gamma(\mu) \sum_{\ell_{3}=0}^{\infty} \frac{\left(k-\frac{m-n}{2}+1\right){ }_{\ell_{3}} \Gamma\left(k+\frac{m+n}{2}+1+\ell_{3}\right)}{\Gamma\left(2 k+2+\ell_{3}\right) \ell_{3} !} \\
& (z-a)^{\lambda+\mu+\ell_{3}+k}(g z+f)^{\delta} \sum_{k_{1}=0}^{\left[\mathrm{V}_{1} / \mathrm{U}_{1}\right]}\left(-\mathrm{V}_{1}\right)_{\mathrm{U}_{1} k_{1}} \ldots\left(-\mathrm{V}_{r}\right)_{\mathrm{U}_{r} \mathrm{k}_{r}} \mathrm{~A}\left[\mathrm{~V}_{1}, k_{1} ; \ldots ; \mathrm{V}_{r}, k_{r}\right]
\end{aligned}
$$




$$
\begin{aligned}
& \prod_{l=1}^{r}\left\{\frac{y_{l}^{k_{l}}(z-a)^{\sigma_{l} k_{l}}}{k_{l} !(a c+d)^{\lambda_{1} k_{l}}(g z+f)^{\mu_{l} k_{l}}}\right\} \sum_{\ell_{1}, \ell_{2}=0}^{\infty} \frac{(-1)^{\ell_{1}}(\mu)_{\ell_{2}}}{\ell_{1} ! \ell_{2} !}\left(\frac{c(z-a)}{a c+d}\right)^{\ell_{1}}\left(\frac{g(z-d)}{g z+f}\right)^{\ell_{2}}
\end{aligned}
$$

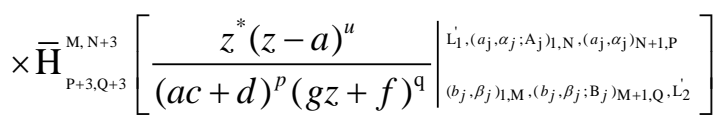

where

$$
\begin{aligned}
& \mathrm{L}_{1}^{\prime}=\left(1+\gamma-\ell_{1}-\Sigma \lambda_{l} k_{l}, p ; 1\right),\left(-\lambda-k-\ell_{1}-\ell_{3}-\Sigma \sigma_{l} k_{l}, u ; 1\right),\left(1+\delta-\ell_{2}-\Sigma \mu_{l} k_{l}, q ; 1\right) \\
& \mathrm{L}_{2}^{\prime}=\left(1+\delta-\Sigma \mu_{l} k_{l}, q ; 1\right),\left(1+\gamma-\Sigma \lambda_{l} k_{l}, p ; 1\right),\left(1-\lambda-\mu-k-\ell_{1}-\ell_{2}-\ell_{3}-\Sigma \sigma_{l} k_{l}, u ; 1\right)
\end{aligned}
$$

(ii) Taking $b=z, \eta=v=0$ in Eq (3.10), the Riemann-Liouville fractional calculus operator of order $-\mu$ of a unified function is obtained as

$$
\begin{aligned}
& { }_{a} \mathrm{D}_{z}^{-\mu}\left\{\frac{(z-a)^{\lambda-1}(c z+d)^{\gamma}(g+z f)^{\delta}}{(z-a-1)^{\frac{n}{2}}} \mathrm{~S}_{n}^{\alpha, \beta, \tau}\left[y \frac{(z-a)^{\sigma}}{(c z+d)^{\zeta}(g z+1)^{\nu}}\right]\right. \\
& \left.\overline{\mathrm{H}}_{\mathrm{P}, \mathrm{Q}, \mathrm{N}}^{\mathrm{M}}\left[\mathrm{z}^{*} \frac{(z-a)^{u}}{(c z+d)^{p}(g z+f)^{q}}\right] Q_{k}^{m, n}\left(1-\frac{2}{z-a}\right)\right\} \\
& =e^{m \pi i} 2^{-\left(\frac{m-n}{2}+1\right)}(-1)^{-\left(k+\frac{n}{2}+1\right)} \Gamma\left(k+\frac{m-n}{2}+1\right) \Gamma(\mu)(z-a)^{\lambda+k+\mu}(a c+d)^{\gamma}(g z+f)^{\delta} \\
& \sum_{b_{1}, b_{2}, a_{1}, a_{2}} \sum_{\ell_{1}, \ell_{2}, \ell_{3}, \ell_{4}=0}^{\infty} \frac{\theta\left(b_{1}, b_{2}, a_{1}, a_{2}\right) y^{\mathrm{R}+\ell_{1}} \tau^{\ell_{1}}\left(a_{1}-s n\right)_{\ell_{1}}}{\ell_{1} ! \ell_{2} ! \ell_{3} ! \ell_{4} !} \\
& \frac{(-1)^{\ell_{2}}(z-a)^{\ell_{3}+\left(\mathrm{R}+t \ell_{1}\right) \sigma}(\mu)_{\ell_{4}}}{(a c+d)^{\left(\mathrm{R}+\ell_{1}\right) \zeta}(g z+f)^{\left(\mathrm{R}+t \ell_{1}\right) v}}\left(\frac{c(z-a)}{a c+d}\right)^{\ell_{2}}\left(\frac{g(z-a)}{g z+f}\right)^{\ell_{4}}
\end{aligned}
$$

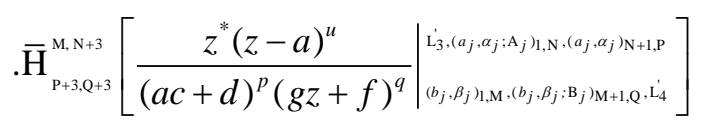

where

$\mathrm{L}_{3}^{\prime}=\left(1+\gamma-\ell_{2}-\left(\mathrm{R}+t \ell_{1}\right) \zeta, p ; 1\right),\left(1+\delta-\ell_{4}-\left(\mathrm{R}+t \ell_{1}\right) v, q ; 1\right),\left(-\lambda-k-\ell_{2}-\ell_{3}-\left(\mathrm{R}+t \ell_{1}\right) \sigma, u ; 1\right)$

$\mathrm{L}_{4}^{\prime}=\left(1+\gamma-\left(\mathrm{R}+t \ell_{1}\right) \zeta, p ; 1\right),\left(1+\delta-\left(\mathrm{R}+t \ell_{1}\right) v, q ; 1\right),\left(-\lambda-\mu-k-\ell_{2}-\ell_{3}-\ell_{4}-\left(\mathrm{R}+t \ell_{1}\right) \sigma, u ; 1\right)$

and other symbols $\theta\left(b_{1}, b_{2}, a_{1}, a_{2}\right), \mathrm{R}$ and $\sum_{b_{1}, b_{2}, a_{1}, a_{2}}$ are same as given in Eqs(2.7), (2.8) and (2.9) respectively.

The conditions of validity of Eqs(5.1) and (5.2) can be obtained from those stated with (3.1) and (3.10).

Riemann-Liouville fractional calculus operator for the results given by (3.7) and (3.13) can also be obtained in similar manner as discussed above. 


\section{IV.CONCLUSION}

In this paper we establish four unified finite integrals involving the product of a general class of a multivariable polynomials, $\bar{H}$ function, generalized associated Legendre function of second kind and generalized polynomial set. A number of several other integrals can also be obtained as special cases of our main results. Since functions and polynomials occurring in these integrals are general in nature, these results can be extended further to provide interesting unifications and extensions of a large number of new and known results.

\section{REFERENCES}

[1] Garg M, Mittal S, "On a new unified integral," in Proceedings Indian Academy of Sciences- Mathematical Sciences, India, 2004.

[2] Ali S, "On some new unified integrals," Adv. Comput. Math Appl., vol. 3, no. 1, pp. 151-153, 2012.

[3] Junesang Cho, Praveen Agarwal, "Certain unified integrals associated with Bessel functions," Boundary Value Problems, 2013.

[4] P Agarwal, "New unified integral involving a Srivastava polynomials and bar H function," Journal of Fractional Calculus and Applications, vol. 3, no. 3, pp. 1-7, 2012.

[5] Amit Chouhan, Arif M. Khan, "Unified Integrals Associated with H-Functions and M-Series," Journal of Fractional Calculus and Applications, vol. 6, no. 2, pp. 11-17, 2015.

[6] Miller, K.S. and Ross, B., An introduction to the Fractional Calculus and Fractional Differential Equation, New York: John Wiley and Sons, (1993).

[7] Inayat-Hussain, A.A., "New properties of hypergeometric series derivable from Feynman integrals II; A generalization of the H-function," $J$. Phys. A: Math. Gen. 20, pp. 4119-4128, 1987.

[8] Srivastava, H.M. , "A multilinear generating function for the Konhauser sets of biorthogonal polynomials suggested by the Laguerre polynomials," Pacific J. Math., vol. 17, pp. 183-191, 1985.

[9] Raizada, A.K. , A Study of Unified Representation of Special Functions of Mathematical Physics and Their Use in Statistical and Boundary Value Problems, Ph.D. Thesis, Bundelkhand University, India, 1991.

[10] L. Kuipers, B. Meulenbeld, "On the generalization of Legendre's associated differential equation," Proc. Konkl. Nederl. Akad. Wet. A. 60,, vol. 60, no. 4, pp. 436-450., 1957. 